\title{
OPEN BK virus infection and outcome following kidney transplantation in childhood
}

\begin{abstract}
James McCaffrey ${ }^{1 \bowtie}$, Vijesh J. Bhute ${ }^{2} \&$ Mohan Shenoy ${ }^{3}$
BK virus associated nephropathy (BKN) is an important cause of kidney allograft failure. In a cohort of paediatric kidney transplant recipients, we aimed to understand the incidence and clinical outcome associated with BKN, as well as identify risk factors for BKN and BK viraemia development. We retrospectively analysed all patients who received a kidney transplant and received follow up care in our centre between 2009-2019. Among 106 patients included in the study (mean follow up 4.5 years), $32 / 106$ (30.2\%) patients experienced BK viraemia. The incidence of BKN was 7/106 (6.6\%). The median time of BK viraemia development post-transplant was 279.5 days compared to 90.0 days for BKN. Development of BKN was associated with younger age at transplantation $(p=0.013)$. Development of BK viraemia was associated with negative recipient serology for cytomegalovirus (CMV) at time of transplantation $(p=0.012)$ and a higher net level of immunosuppression $(p=0.039)$. There was no difference in graft function at latest follow up between those who experienced BKN and those without BKN. This study demonstrates that BK virus infection is associated with younger age at transplantation, CMV negative recipient serostatus and higher levels of immunosuppression. Judicious monitoring of BK viraemia in paediatric transplant recipients, coupled with timely clinical intervention can result in similar long-term outcomes for BKN patients compared to controls.
\end{abstract}

$\mathrm{BK}$ virus $(\mathrm{BKPyV})$ is a member of the Polyomaviridae family of double-stranded DNA viruses ${ }^{1}$. Primary $\mathrm{BKPyV}$ infection is mainly asymptomatic and occurs predominantly before adolescence, with an IgG seroprevalence $>90 \%$ in 5-9 year old healthy children. Seroprevalence rates in later life demonstrate an age-dependent decline with $68 \%$ of $60-69$ year olds showing IgG positivity ${ }^{2}$. Peripheral blood mononuclear cells disseminate $\mathrm{BKPyV}$ to the urinary tract where the virus establishes a persistent non-replicative latent phase in renal tubular epithelial cells and urothelium ${ }^{3,4}$. Periodic BKPyV reactivation occurs, with asymptomatic urinary shedding seen in $7 \%$ of healthy adults ${ }^{5}$.

In kidney allograft recipients, active replication of $\mathrm{BKPyV}$ can lead to $\mathrm{BKPyV}$-associated nephropathy (BKN) and subsequent graft dysfunction and premature loss ${ }^{6}$. The emergence of BKN in the last decade of the twentieth century coincided with the introduction of potent immunosuppressive agents such as tacrolimus and mycophenolate mofetil (MMF), leading to the proposal that a higher level of immunosuppression is a risk factor for the development of $\mathrm{BKN}^{7,8}$. $\mathrm{BKPyV}$ viraemia precedes the onset of $\mathrm{BKN}$, and polymerase-chain-reaction (PCR) assays of plasma are a specific and sensitive method to detect early nephropathy ${ }^{9}$. Before recognition of the necessity to identify and treat BKN in a timely manner, graft loss rates as high as $67 \%$ were documented in the adult population ${ }^{10}$.

Anti-viral agents have demonstrated minimal or no efficacy in clearing $\mathrm{BKPyV}^{11-13}$, and treatment involves reducing immunosuppressive therapy to restore the capability of the host immune system to control BKPyV replication and prevent progression to $\mathrm{BKN}^{14}$. Levels of $\mathrm{BKPyV}$-specific IgG antibodies and T-cells increase following a reduction in immunosuppression and peak at the time of BKPyV viraemia resolution ${ }^{15}$.

In the paediatric kidney transplant population, rates of $\mathrm{BKPyV}$ viraemia range from $18-37 \%$ and $\mathrm{BKN}$ is identified in $0-16 \%$ of patients ${ }^{8,15-23}$. In a case series of 32 patients under the age of 20 years with BKN, $3(9 \%)$ of allografts were lost ${ }^{6}$. Reported risk factors for the development of BKN in the paediatric population include allograft recipient BKPyV seronegativity at time of transplantation ${ }^{20,23}$, zero human leukocyte antigens (HLA) -A and -DR mismatches between transplant donor and recipient ${ }^{22}$, increased levels of immunosuppression, ${ }^{8,21}$

\footnotetext{
${ }^{1}$ Department of Histopathology, Cambridge University Hospitals NHS Foundation Trust, Addenbrooke's Hospital, Cambridge, UK. 'Department of Chemical Engineering, Imperial College London, South Kensington Campus, London SW7 2AZ, UK. 3 Department of Paediatric Nephrology, Royal Manchester Children's Hospital, Manchester University Hospitals NHS Foundation Trust, Manchester Academic Health Science Centre, Manchester, UK. ${ }^{\boxplus e m a i l:}$ jm2199@cam.ac.uk
} 


\begin{tabular}{|c|c|c|c|c|c|c|c|}
\hline \multirow[b]{2}{*}{ Characteristic } & \multicolumn{2}{|l|}{ No-BKV $(n=74)$} & \multicolumn{2}{|l|}{$\operatorname{BKN}_{\text {Low }}(\mathbf{n}=25)$} & \multicolumn{2}{|l|}{$\mathrm{BKN}_{\mathrm{Hi} / \mathbf{B}+}(\mathbf{n}=7)$} & \multirow[b]{2}{*}{$p$ value } \\
\hline & Proportion (\%) & Mean & Proportion (\%) & Mean & Proportion (\%) & Mean & \\
\hline Female & $24 / 74(32.4 \%)$ & & $9 / 25(36.0 \%)$ & & $2 / 7(28.6 \%)$ & & \multirow{2}{*}{0.939} \\
\hline Male & $50 / 74(67.6 \%)$ & & $16 / 25(64.0 \%)$ & & $5 / 7(71.4 \%)$ & & \\
\hline Age at transplant (years) & & 11.2 & & 10.2 & & 6.2 & 0.0369 \\
\hline Ethnicity: White British ${ }^{+}$ & $45 / 68(66.2 \%)$ & & $17 / 25(68.0 \%)$ & & $5 / 7(71.4 \%)$ & & 1.000 \\
\hline Living transplant donor & $43 / 74(58.1 \%)$ & & $18 / 25(72.0 \%)$ & & $6 / 7(85.7 \%)$ & & 0.228 \\
\hline Cold ischaemia time (minutes) & & 345.2 & & 235.6 & & 143.9 & 0.218 \\
\hline Steroid-free regimen at latest follow up & $44 / 74(59.5 \%)$ & & $11 / 25(44.0 \%)$ & & $4 / 7(57.1 \%)$ & & 0.448 \\
\hline MMF part of regimen at latest follow up & $55 / 74(74.3 \%)$ & & $16 / 25(64.0 \%)$ & & $5 / 7(71.4 \%)$ & & 0.543 \\
\hline Exposure to anti-CMV therapy & $24 / 74(32.4)$ & & $10 / 25(40.0 \%)$ & & $3 / 7(42.9 \%)$ & & 0.735 \\
\hline BPAR & $21 / 74(28.4)$ & & $6 / 25(24.0 \%)$ & & $2 / 7(28.6 \%)$ & & 0.933 \\
\hline Graft failure & 7/74 (9.5\%) & & $1 / 25(4.0 \%)$ & & $0 / 7(0.0 \%)$ & & 0.802 \\
\hline Death & $2 / 74(2.7 \%)$ & & $0 / 25(0.0 \%)$ & & $0 / 7(0.0 \%)$ & & 1.000 \\
\hline Length of follow up (months) & 55.8 & & 48.0 & & 60.1 & & 0.453 \\
\hline
\end{tabular}

Table 1. Univariate analysis of patient cohort characteristics. ${ }^{+}$Data not available for six patients. $\mathrm{BPAR}=$ biopsy proven acute rejection.

younger age of recipient at transplantation ${ }^{21}$, and a tacrolimus- compared to ciclosporin- based immunosuppression regimen ${ }^{21}$. A recent study in adult kidney transplant recipients suggested prophylactic use of the anticytomegalovirus (CMV) agent valganciclovir may be associated with an increased risk of $\mathrm{BKN}^{24}$.

In this single-centre study, we report our experience of BKN in a paediatric transplant recipient population over a 10-year period, with patients predominantly receiving a steroid-sparing immunosuppressive regimen ${ }^{25}$. Specific aims of the study were to: (1) evaluate rates of BKPyV viraemia and BVN in this population; (2) understand the morbidity associated with BKPyV viraemia and BKN; and (3) identify risk factors for the development of BKPyV viraemia and BKN.

\section{Results}

Study population. A total of 106 patients met the requirements for inclusion in the study, with a mean follow up time of 54.3 months. There were 7 graft failures (mean time in months post-transplant: 44.0 months, range 18-70 months) and 2 deaths (see Supplementary Table S1). There were no graft losses or deaths in $\mathrm{BKN}_{\mathrm{Hi} / \mathrm{B}+}$ patients (Table 1). 99/106 patients received the steroid-sparing TWIST immunosuppressive regimen. 7/106 patients received a non-TWIST immunosuppression regimen (reasons including enrolment in research trials and transplant occurring before TWIST became standard local protocol). Characteristics for the whole cohort are provided in Supplementary Tables S2, S3 and S4.

Incidence of BKN. There were 41 episodes of BK viraemia in 32/106 patients (30.2\%). 2/106 patients (1.9\%) experienced an episode of $\mathrm{BKN}_{\mathrm{B}+}$, and a further $5 / 106(4.7 \%)$ patients experienced an episode of $\mathrm{BKN}_{\mathrm{Hi}}$. Therefore, the incidence of $\mathrm{BKN}_{\mathrm{Hi} / \mathrm{B}+}$ was $7 / 106(6.6 \%) .25 / 106$ patients $(23.6 \%)$ experienced an episode of $\mathrm{BKN}_{\text {Low }}$ without an episode of $\mathrm{BKN}_{\mathrm{Hi} / \mathrm{B}+}$ at any point post-transplantation.

Timing of episodes. The mean (median) time of viraemia onset post-transplant in the 34 episodes of $\mathrm{BKN}_{\text {Low }}$ was 508.4 (279.5) days compared to 234.3 (90.0) days for $\mathrm{BKN}_{\mathrm{Hi} / \mathrm{B}+}(p=0.199)$ (Fig. 1a and Supplementary Table S5). The range of time of onset of viraemia post-transplant was 19-1775 days for $\mathrm{BKN}_{\mathrm{Low}}$ episodes and 33-656 days for $\mathrm{BKN}_{\mathrm{Hi} / \mathrm{B}+} \cdot 23.5 \%$ of $\mathrm{BKN}_{\mathrm{Low}}$ episodes occurred more than 2 years post transplant. In contrast, all episodes of $\mathrm{BKN}_{\mathrm{Hi} / \mathrm{B}+}$ occurred within the first 2 years following transplantation.

$12 / 34(35.3 \%)$ of total $\mathrm{BKN}_{\text {Low }}$ episodes occurred in the 6 months immediately post-transplant compared to $5 / 7(71.4 \%)$ episodes of $\mathrm{BKN}_{\mathrm{Hi} / \mathrm{B}+}$. A plot of the Kaplan-Meier estimator demonstrated no significant difference between the timing of onset of $\mathrm{BKN}_{\mathrm{Low}}$ and $\mathrm{BKN}_{\mathrm{Hi} / \mathrm{B}+}$ viraemia (Fig. 1b). An overview of the temporal distribution of BK viraemia episodes is provided in Fig. 2.

No difference between length of viraemia per episode was noted between $\mathrm{BKV}_{\mathrm{All}}$ subgroups (mean 105.8 days for $\mathrm{BKN}_{\mathrm{Low}}$ compared to 104.0 days for $\mathrm{BKN}_{\mathrm{Hi} / \mathrm{B}+}, p=0.265$ ) (Fig. 1c and Supplementary Table S6).

Analysis of risk factors. Univariate analysis was performed to assess potential risk factors for the development of BKPyV viraemia and BKN. Age at transplant was significantly different between the 3 groups $(p=0.0369)$ (Table 1). Bonferroni's post-test was used to compare groups and there was a significant difference in age at transplantation between no-BKV patients (mean 11.2 years) compared to $\mathrm{BKN}_{\mathrm{Hi} / \mathrm{B}+}$ patients (mean 6.2 years) ( $p=0.013$, adjusted critical value $\alpha_{\text {Bonferroni }}=0.0167$ for three group comparison). There was no difference between age at transplantation between no-BKV patients and $\mathrm{BKN}_{\text {Low }}$ patients (mean 10.2 years) $(p=0.363)$.

CMV serology status of allograft recipients was also significantly different between the groups $(p=0.012)$

(Table 2). $\mathrm{BKN}_{\text {Low }}$ patients were less likely to have recorded positive CMV serology at time of transplantation 
a
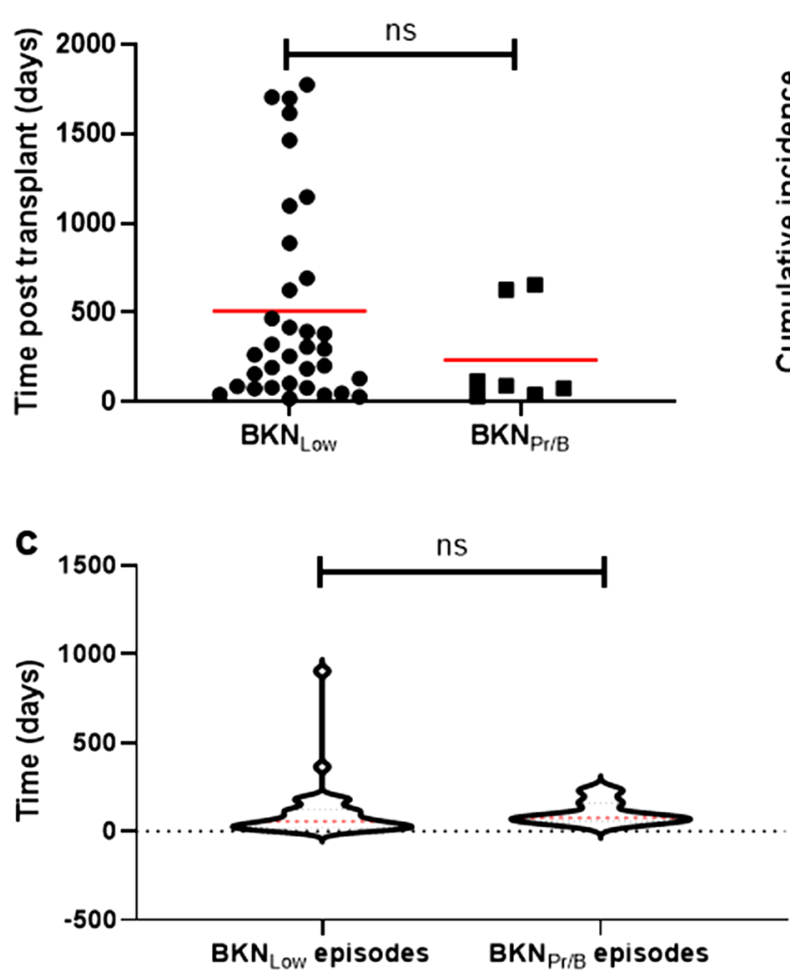

b

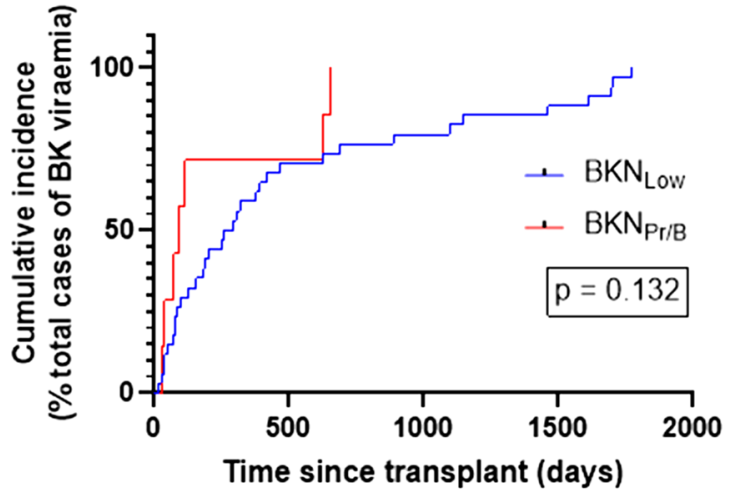

Figure 1. Characteristics of BKyPV viraemia episodes. (a) Time of onset of viraemia episodes post-transplant ( $p=0.199$, Mann-Whitney U test). Red line indicates mean value for each group. (b) Cumulative risk of developing BKPyV viraemia over time (curves compared using log-rank / Mantel-Cox test). (c) Violin plot comparing length of viraemia episodes between groups ( $p=0.265$, Mann-Whitney U test). Quartiles are plotted with median value in red. ns= not significant.

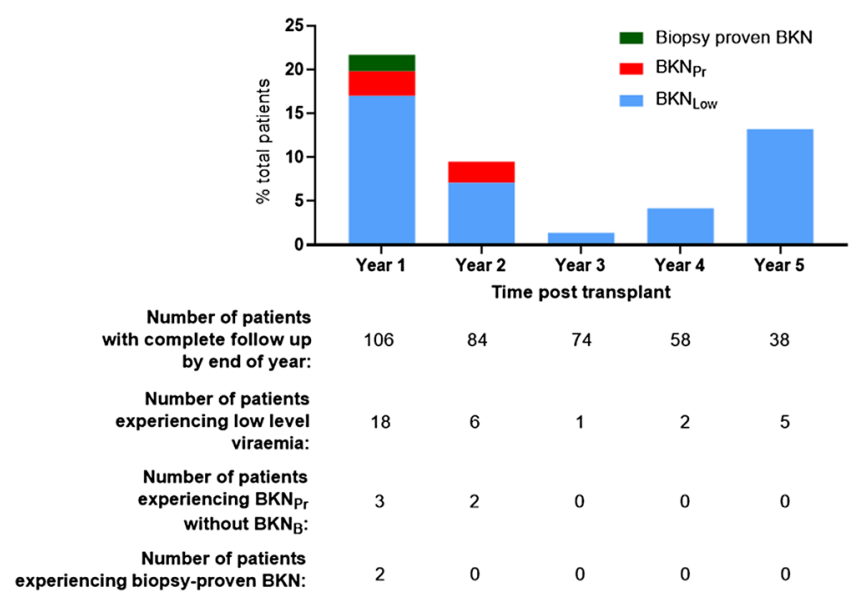

Figure 2. Incidence of $\mathrm{BKN}_{\mathrm{Low}}, \mathrm{BKN}_{\mathrm{B}+}$ and $\mathrm{BKN}_{\mathrm{Hi}}$ in years 1-5 post transplantation.

\begin{tabular}{|l|l|l|l|l|l|}
\hline & & $\begin{array}{l}\text { No BK } \\
\text { Proportion (\%) }\end{array}$ & $\begin{array}{l}\mathbf{B K N}_{\text {Low }} \\
\text { Proportion (\%) }\end{array}$ & $\begin{array}{l}\mathbf{B K N}_{\text {Hi/B+ }} \\
\text { Proportion (\%) }\end{array}$ & $\boldsymbol{p}$ value \\
\hline \multirow{2}{*}{ CMV status donor } & + & $29 / 74(39.2 \%)$ & $11 / 25(44.0 \%)$ & $3 / 7(42.9 \%)$ & \multirow{2}{*}{0.944} \\
\cline { 2 - 5 } & - & $45 / 74(60.8 \%)$ & $14 / 25(56.0 \%)$ & $4 / 7(57.1 \%)$ & \\
\hline \multirow{2}{*}{ CMV status recipient } & + & $27 / 74(36.5 \%)$ & $2 / 25(8.0 \%)$ & $3 / 7(42.9 \%)$ & \multirow{2}{*}{0.012} \\
\cline { 2 - 5 } & - & $47 / 74(63.5 \%)$ & $23 / 25(92.0 \%)$ & $4 / 7(57.1 \%)$ & \\
\hline
\end{tabular}

Table 2. Univariate analysis of CMV serostatus of transplant donor and recipient. 


\begin{tabular}{|c|c|c|c|c|c|c|c|c|}
\hline \multirow[b]{2}{*}{ Factor } & \multirow{2}{*}{$\begin{array}{l}p \text { value of reduced } \\
\text { model }^{\mathrm{a}}\end{array}$} & \multirow{2}{*}{$\begin{array}{l}p \text { value of final } \\
\text { model }\end{array}$} & \multicolumn{3}{|c|}{ BKN $_{\text {Low }}$ status ${ }^{\mathrm{d}}$} & \multicolumn{3}{|c|}{ BKN $_{\mathrm{Hi} / \mathbf{B}+}$ status $^{\mathrm{d}}$} \\
\hline & & & $\mathbf{O R}^{\mathrm{b}}$ & $95 \% \mathrm{CI}^{\mathrm{c}}$ & $p$ value & $\mathrm{OR}^{\mathrm{b}}$ & $95 \% \mathrm{CI}^{\mathrm{c}}$ & $p$ value \\
\hline Age at transplant & 0.027 & \multirow[b]{2}{*}{0.002} & 0.975 & 0.889 to 1.070 & 0.594 & 0.785 & 0.636 to 0.968 & 0.024 \\
\hline $\begin{array}{l}\text { CMV serostatus of } \\
\text { recipient }\end{array}$ & 0.009 & & 0.157 & 0.034 to 0.723 & 0.018 & 1.869 & 0.350 to 9.968 & 0.464 \\
\hline
\end{tabular}

Table 3. Multinomial Logistic Regression Analysis. ${ }^{a}$ Log Likelihood tests ; ${ }^{b} \mathrm{OR}$ : odds ratio; ${ }^{\mathrm{c}} \mathrm{CI}$ : confidence interval for odds ratio; ${ }^{\mathrm{d}}$ Reference category is No-BKV.

a

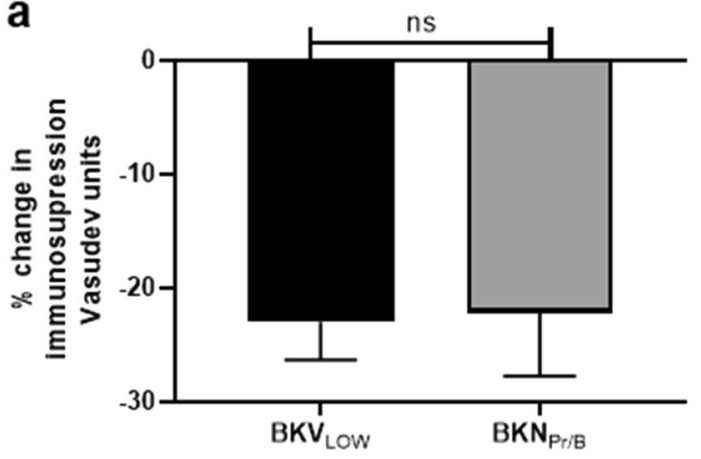

C

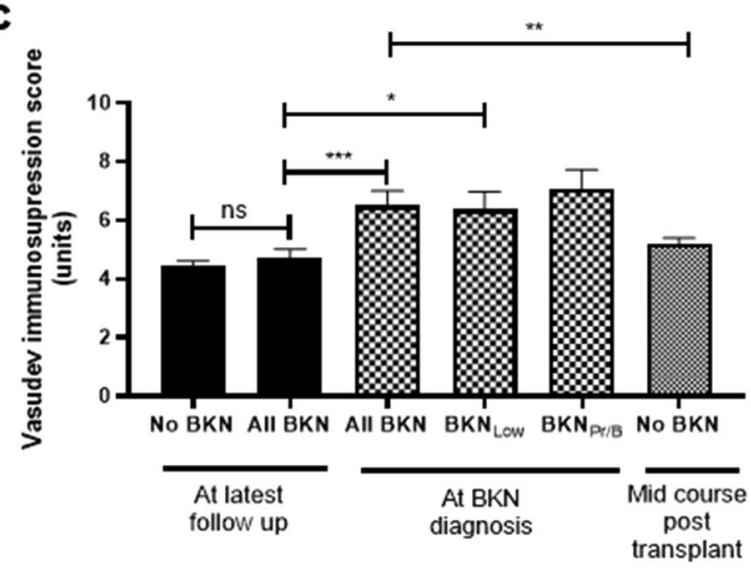

b

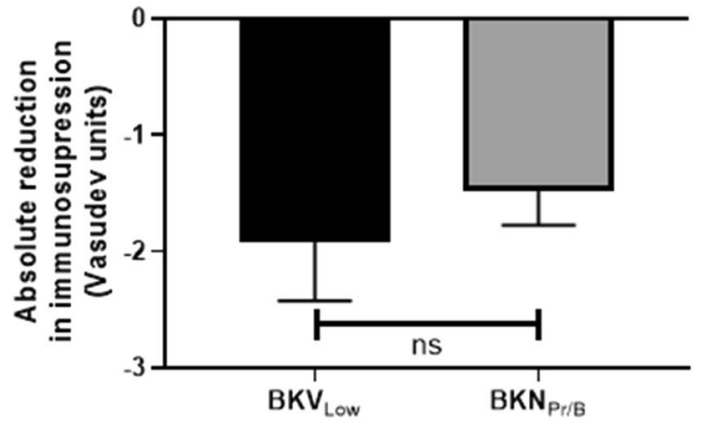

Figure 3. Change in net immunosuppression over time. (a) Percentage change in paediatric immunosuppression Vasudev score during episodes of BKPyV viraemia ( $p=0.588$, Mann-Whitney U test). (b) Absolute change in paediatric immunosuppression Vasudev score during episodes of BKPyV viraemia ( $p>0.999$, Mann-Whitney U test). c Mean paediatric Vasudev immunosuppression score at latest follow up, at $\mathrm{BKPyV}$ viraemia diagnosis and at 462 days post-transplantation in no-BKV group. Groups compared using one way ANOVA followed by Tukey's test. ns $=$ not significant $(p=0.996),{ }^{* *} p=0.0115,{ }^{* *} p=0.0390,{ }^{*} p=0.0391$.

$(2 / 25,8.0 \%)$ than no-BKV (27/74. 36.5\%) and $\mathrm{BKN}_{\mathrm{Hi} / \mathrm{B}+}(3 / 7,42.9 \%)$ patients. There was no difference in exposure to anti-CMV therapy between patient sub-groups (Table 1 and Supplementary Table S7).

No differences between patient subgroups were identified for the following characteristics: sex, cold ischaemia time, ethnic background, frequency of BPAR, use of steroids/MMF, source of graft donor (living/cadaveric) and HLA mismatch status (Table 1 and Supplementary Table S8).

Multinomial logistic analysis confirmed the univariate analysis findings of CMV recipient serostatus and age at time of transplant being independently predictive of BKN and BKPyV viraemia. Additionally, age and CMV serostatus in the same model are predictive of BKN/ BKPyV viraemia $(p=0.002)$ (Table 3$)$.

Immunosuppression. All (7/7) episodes of $\mathrm{BKN}_{\mathrm{Hi} / \mathrm{B}+}$ elicited a reduction in immunosuppression by the treating clinician compared to $13 / 34(38.2 \%)$ of episodes of $\mathrm{BKN}_{\text {Low }}$ (Supplementary Table S9). However, when the decision to reduce immunosuppression was made, there was no significant difference between the size of reduction between $\mathrm{BKN}_{\mathrm{Low}}$ (mean reduction in paediatric Vasudev score-23.0\%) and $\mathrm{BKN}_{\mathrm{Hi} / \mathrm{B}+}$ (mean reduction in paediatric Vasudev score -22.2\%) (Fig. 3a,b).

To understand how the total level of immunosuppression varied for patients across their post-transplant clinical course, a paediatric Vasudev score was calculated for all patients at latest follow up and, for relevant patients, at time of BKPyV viraemia onset. Additionally we quantified the level of immunosuppression for 
a

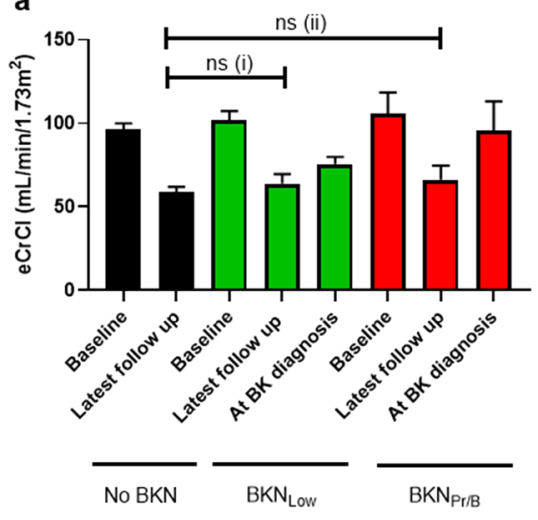

c

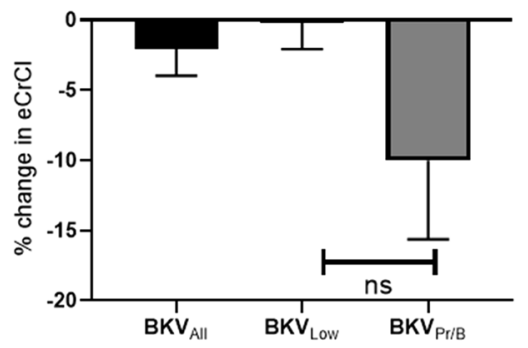

b
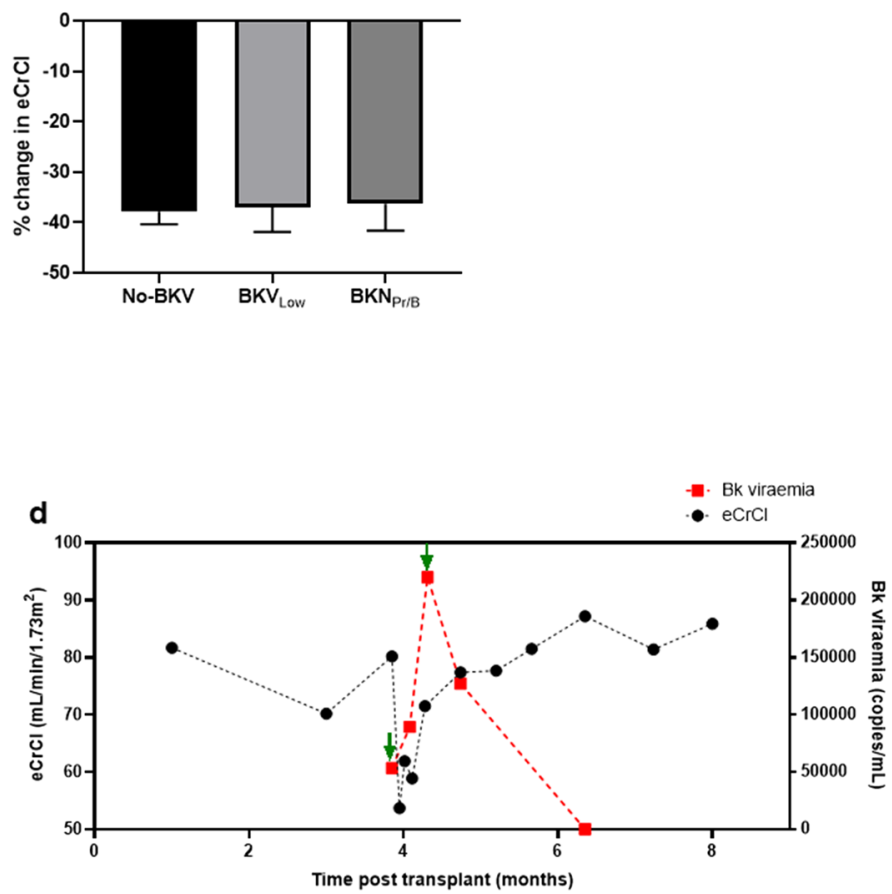

Figure 4. Effect of $\mathrm{BKPyV}$ viraemia on graft function. (a) Estimated creatinine clearance $(\mathrm{eCrCl})$ at baseline, latest follow up and at $\mathrm{BKPyV}$ viraemia diagnosis. Black bars = 'No-BKV', green bars $=$ ' $\mathrm{BKN} \mathrm{Low}_{\mathrm{Lw}}$, red bars $={ }^{' B K N} \mathrm{BK}_{\mathrm{Hi} / \mathrm{B}+}$. [ns (1) $p=0.996$, ns (2) $\mathrm{p}=0.998$, one way ANOVA followed by Tukey's test]. (b) Percentage change in eCrCl at latest follow up compared to baseline. (c) Percentage change in lowest eCrCl recorded during $\mathrm{BKPyV}$ viraemia compared to $\mathrm{eCrCl}$ at $\mathrm{BKPyV}$ viraemia diagnosis (ns $p=0.109$, Mann-Whitney $\mathrm{U}$ test). (d) Representative plot showing plasma viral load and $\mathrm{eCrCl}$ of a patient with $\mathrm{BKN}_{\mathrm{Hi}}$ over time. Green arrows indicate points at which immunosuppressive therapy was reduced.

no-BKV patients at the mean time $\mathrm{BKV}_{\mathrm{All}}$ patients developed viraemia post-transplant (462 days). This allowed us to analyse no-BKV and $\mathrm{BKV}_{\text {All }}$ patients at comparable points in their clinical course.

There was no significant difference in paediatric Vasudev score at latest follow up between no-BKV patients (mean 4.4 units) compared to $\mathrm{BKV}_{\mathrm{All}}$ patients (mean 4.7 units) (Fig. 3c). However, the level of immunosuppression was significantly higher for $\mathrm{BKV}_{\text {All }}$ patients at time of $\mathrm{BKPyV}$ viraemia diagnosis (mean 6.5 units) compared to latest follow-up ( $p=0.012$ ). Furthermore, immunosuppression level in no-BKV patients at 462 days post-transplantation (mean 5.2 units) was significantly lower than for $\mathrm{BKV}_{\mathrm{All}}$ patients at $\mathrm{BKPyV}$ viraemia diagnosis $(p=0.0390)$. This shows that patients who developed BKPyV viraemia were exposed to a higher level of immunosuppression at time of diagnosis than patients without $\mathrm{BKPyV}$ viraemia.

To understand if increases in immunosuppression following an episode of acute rejection may explain the higher paediatric Vasudev score recorded for $\mathrm{BKV}_{\mathrm{All}}$ patients, we examined whether BKPyV viraemia or acute rejection occurred first for each relevant patient. There were 8 episodes of BPAR among $B K V_{\text {All }}$ patients: in 4/8 episodes rejection occurred before $\mathrm{BKPyV}$ viraemia and in 4/8 episodes BKPyV viraemia occurred before rejection. $2 / 2 \mathrm{BKN}_{\mathrm{B}+}$ patients experienced an episode of acute rejection: in both instances $\mathrm{BKN}$ occurred before acute rejection.

Effect on kidney function. There was no significant difference in eCrCl between patient sub-groups at latest follow up or significant difference in the eCrCl percentage change from baseline to latest follow up between patient sub-groups (Fig. 4a,b).

To analyse changes in kidney function during periods of viraemia, the $\mathrm{eCrCl}$ at $\mathrm{BKPyV}$ viraemia diagnosis was compared to the lowest eCrCl during the period of viraemia for each patient and a percentage change was calculated. $\mathrm{BKN}_{\text {Low }}$ patients showed a mean eCrCl change of $-0.2 \%$, while $\mathrm{BKN}_{\mathrm{Hi} / \mathrm{B}+}$ patients showed a mean eCrCl change of $-10.0 \%$ (Fig. 4c). However, there was no significant difference between the $\mathrm{eCrCl}$ at $\mathrm{BKPyV}$ viraemia diagnosis and the average $\mathrm{eCrCl}$ reading during viraemia for either $\mathrm{BKN}_{\mathrm{Low}}$ or $\mathrm{BKN}_{\mathrm{Hi} / \mathrm{B}+}$ patients (Supplementary Figure S1). A representative plot of plasma viral load and $\mathrm{eCrCl}$ of a patient with $\mathrm{BKN}_{\mathrm{Hi}}$ is shown in Fig. $4 \mathrm{~d}$.

\section{Discussion}

The salient findings of this study involving 106 paediatric kidney transplant recipients are as follows: (1) 2/106 individuals experienced $\mathrm{BKN}_{\mathrm{B}+}$ and 5/106 patients developed $\mathrm{BKN}_{\mathrm{Hi}}$, giving an overall $\mathrm{BKN}$ incidence of $7 / 106$ (6.6\%); (2) $30.2 \%$ patients experienced $\mathrm{BKPyV}$ viraemia; (3) the incidences of $\mathrm{BKN}_{\mathrm{Low}}, \mathrm{BKN}_{\mathrm{B}+}$ and $\mathrm{BKN}_{\mathrm{Hi}}$ were highest during the first year post-transplant; (4) $23.5 \%$ of $\mathrm{BKN}_{\mathrm{Low}}$ episodes occurred after 2 years post-transplantation; (5) there was no significant difference in long-term graft function between patients experiencing BKPyV 
viraemia/BKN and no-BK controls; (6) younger age at transplantation, CMV negative serology of the allograft recipient at time of transplantation, and a higher level of immunosuppression were associated with an increased risk of $\mathrm{BKN} / \mathrm{BKPyV}$ viraemia development.

Our incidence of $\mathrm{BKN}_{\mathrm{B}+}(1.9 \%)$ is slightly lower than rates of 3.5-4.5\% recorded in previous studies ${ }^{20-22}$. We found similar levels of BKPyV viraemia to a recent European mulitcentre study (30.2\% vs. 36.7\%), but lower level of $\mathrm{BKN}_{\mathrm{Hi}}(4.7 \% \text { vs. } 15.8 \%)^{21}$. There were no graft losses from $\mathrm{BKN}_{\mathrm{Hi} / \mathrm{B}+}$ patients in our cohort, whereas graft failure rates of $7.1-24.0 \%$ have previously been recorded in American and European data ${ }^{21,22}$.

There was no difference in $\mathrm{eCrCl}$ at final follow up between patients experiencing $\mathrm{BKPyV}$ viraemia or $\mathrm{BKN}$ and patients without BKPyV viraemia / BKN. Our graft outcome findings are similar to a prospective study of 62 paediatric transplants which reported no difference in SCr between patients who developed BKPyV viraemia and those who did not (36 months follow up post-transplantation $)^{15}$. Data from adult transplant recipients indicated that immunosuppression can safely be reduced in patients with $\mathrm{BKPyV}$ viraemia to prevent progression to $\mathrm{BKN}$, without a concomitant increase in the incidence of acute rejection ${ }^{14}$. However, some paediatric data suggested an increased risk of $\mathrm{BKPyV}$ viraemia in patients who had experienced an episode of acute rejection ${ }^{15}$. In the current study, we found no difference in rates of BPAR among patient sub-groups, and in the 8 episodes of BPAR occurring in $\mathrm{BKV}_{\mathrm{All}}$ patients, only half preceded $\mathrm{BKPyV}$ viraemia development. These observations suggest that increases in immunosuppression due to rejection are unlikely to be a major contributor to $\mathrm{BKPyV}$ viraemia development in our cohort.

Rates of BKPyV viraemia in the current study exceed those found in the adult transplant population (11.5-12.8\% $)^{14,26}$, and suggests children may have an increased risk of developing BKPyV-associated pathology. Indeed, we identified younger age at transplantation as a risk factor for BKN development. As BKPyV seropositivity increases from $6 \%$ in the $1-4$ year age range to $91 \%$ in the 5-9 year age range ${ }^{26}$, it is likely that a proportion of the BKN episodes in our cohort among the younger children represented primary BKPyV infection. No definitive evidence exists demonstrating that primary BKPyV replication in seronegative individuals results in a poorer clinical outcome compared to secondary BKPyV replication in seropositive individuals ${ }^{6}$. However, our finding is in agreement with other studies ${ }^{21}$, and suggests younger transplant recipients may benefit from increased frequency of monitoring and rapid instigation of treatment to prevent uncontrolled viral replication.

We found that CMV seronegative individuals were more likely to develop BKPyV viraemia than CMV seropositive patients. Following a recent report linking prophylactic valganciclovir use with an increased risk of $\mathrm{BKN}^{24}$, we investigated whether anti-CMV therapy (routinely prescribed in CMV negative individuals) was associated with an increased risk of BKPyV replication in our cohort. However, we found no evidence to support this (Table 1 and Supplementary Table S7). CMV seropositivity rapidly increases with age during childhood in a similar manner to rates of $\mathrm{BKPyV}$ seropositivity ${ }^{27}$. It is therefore possible that seronegative CMV individuals are more likely to also be $\mathrm{BKPyV}$ seronegative, and therefore at heightened risk of uncontrolled viral replication during primary $\mathrm{BKPyV}$ infection. However, due to the retrospective nature of the study, this conjecture cannot be confirmed.

Our data supports previous observations that a higher level of immunosuppression is a risk factor for $\mathrm{BKPyV}$ reactivation. A tacrolimus/MMF-based immunosuppression regimen has been shown to double the risk of $\mathrm{BKPyV}$ viraemia compared to a ciclosporin /MMF-based regimen ${ }^{21}$. However, rates of BKPyV viraemia in our cohort (predominantly tac/MMF-based regimen) are comparable to those treated with alternative regimens.

Limitations of the current study include the retrospective approach and relatively small number of subjects in comparison to multi-centre investigations. In particular, the low numbers of patients experiencing BKN in this study reduces the confidence with which definitive conclusions can be drawn from these data. Additionally, a prospective approach would have allowed collection of biological samples at various time points throughout a patient's clinical course to interrogate $\mathrm{BKPyV}$ serostatus at the time of transplantation and understand the effect seronegativity has on clinical outcome.

Overall, our results demonstrate that control of $\mathrm{BKPyV}$ replication in paediatric transplant recipients can be attained through judicious surveillance and timely reduction in immunosuppression, without an increased risk of acute rejection. This approach results in comparable graft function and survival outcomes between patients developing $\mathrm{BKN}$ and those without evidence of viraemia. The findings that younger age at transplantation significantly increases a patient's risk of developing BKN, and almost a quarter of BKPyV viraemia episodes occur later than 2 years post-transplant has implications for the development of BKN surveillance strategies.

\section{Methods}

Study design. This is a retrospective cohort analysis of patients receiving a kidney transplant at the Royal Manchester Children's Hospital (RMCH) (UK). Case notes were reviewed from June 2009 to January 2019 and patients who met the following requirements were included in the study: (1) Aged < 19 years at time of transplantation; (2) a minimum of 12 months follow-up data; and (3) transplant performed at RMCH. For patients who received more than one transplant, the first transplant was evaluated.

Clinical and serological data were collected at time of transplant and at months 1, 3, 6, 9, 12 months posttransplantation and at 6 monthly intervals thereafter. All plasma BKPyV quantification and histology results were recorded. Immunosuppression regimens for each patient were documented at latest follow up, and during $\mathrm{BKPyV}$ viraemia episodes for relevant patients. Contemporaneous patient height and weight results were recorded for each serum creatinine ( $\mathrm{SCr}$ ) reading, to allow the estimated creatinine clearance (eCrCl) to be calculated using the standard Schwartz formula $(\mathrm{k}=40 \text {, as per local protocol })^{28}$. All SCr readings during periods of BKPyV viraemia were recorded. The study protocol was reviewed by the Clinical Trial Management Department (Manchester University NHS Foundation Trust) who waived ethical approval for this study involving the retrospective collection of anonymised data. They concluded that informed consent was not required from 
study participants. The study was performed in accordance with Manchester University NHS Foundation Trust guidelines. No patient identifiable data was recorded.

BKPyV surveillance and immunosuppression regimen. Plasma BKPyV monitoring is performed in all kidney transplant recipients every month during the first year post-transplantation and on an annual basis thereafter using a TaqMan PCR-based approach. Monitoring is performed more frequently at the treating clinician's discretion.

The standard local immunosuppression approach is the steroid-sparing (TWIST) protocol ${ }^{25}$. Briefly, this involves induction therapy with the interleukin 2 receptor monoclonal antibody antagonist, basiliximab, on day 0 and day 4 post transplantation, coupled with a rapidly weaning course of prednisolone which is discontinued on day 5. Long-term immunosuppression is provided by tacrolimus and MMF. The net immunosuppressive load at different time points for each patient was quantified using the paediatric Vasudev score ${ }^{29}$. This score provides an estimation of the overall immunosuppressive load by integrating the immunosuppressant types, doses and body surface area of each patient. For example, a patient administered $1.2 \mathrm{mg} / \mathrm{m}^{2}$ per day of tacrolimus (equating to a paediatric Vasudev score of 1 unit) and $580 \mathrm{mg} / \mathrm{m}^{2}$ per day of MMF (equating to a paediatric Vasudev score of 2 units), would have a total paediatric Vasudev score of 3 units.

Definitions. Patients with no recorded positive BKPyV plasma result or histological evidence of BKN were designated as 'no-BKV.' Patients with any recordable level of BKPyV viraemia at any time during study follow-up were designated as ' $\mathrm{BKV} \mathrm{V}_{\mathrm{All}}$ '. The $\mathrm{BKV}_{\mathrm{All}}$ group was subdivided into three groups: (1) those with biopsy-proven $\mathrm{BKN}$ (' $\mathrm{BKN}_{\mathrm{B}+}$ '); (2) those with presumptive $\mathrm{BKN}$ (' $\mathrm{BKN}_{\mathrm{Hi}}$ ') without histological evidence of $\mathrm{BKN}$. BKN $\mathrm{Hi}_{\mathrm{Hi}}$ is defined as a sustained ( $>3$ week) high-level viraemia $\left(>10^{4}\right.$ copies $\left./ \mathrm{mL}\right)$, as per international recommendations ${ }^{21,30}$; and (3) patients with $\mathrm{BKPyV}$ viraemia but not meeting the requirements for either $\mathrm{BKN}_{\mathrm{B}+}$ or $\mathrm{BKN}_{\mathrm{Hi}}\left({ }^{(} \mathrm{BKN} \mathrm{Hi}_{\mathrm{B}+}{ }^{\prime}\right)$, were designated as having low-level viraemia ('BKN $\mathrm{Bow}_{\text {') }}$ ).

The length of viraemia for each episode was defined as the length of time from the first positive BKPyV plasma viraemia result until plasma levels were again undetectable. Baseline eCrCl was defined as the highest $\mathrm{eCrCl}$ recorded during the first year following transplantation. Graft failure was defined as the requirement to return to dialysis after the first week post-transplantation.

Statistical analysis. Univariate analysis of risk factors was performed using Fisher's exact test and chisquared test for correlation (for categorical variables) and one-way analysis of variance (ANOVA) with Bonferroni's post-hoc test (for quantitative variables). Analysis of clinical outcomes was performed using the nonparametric Mann-Whitney U test or one-way ANOVA with Tukey's test, as appropriate.

Multinomial analysis. A multinomial logistic regression analysis was used to predict the BKN development. The following variables were considered for the model: gender, ethnicity, age at transplant, cold ischaemia time, biopsy-proven acute rejection (BPAR), source of allograft (cadaveric/living), use of steroid-free regimen at latest follow-up, use of MMF at latest follow up, exposure to anti-CMV therapy, HLA mismatch, recipient CMV status, donor CMV status. The analysis was performed in IBM SPSS Statistics Version 26. A parsimonious model was developed by using forward selection method. The final model consisted of one factor: CMV serostatus of recipient and one covariate: age at transplant.

Received: 10 November 2020; Accepted: 14 January 2021

Published online: 28 January 2021

\section{References}

1. Johne, R. et al. Taxonomical developments in the family Polyomaviridae. Adv. Virol. 156, 1627-1634 (2011).

2. Knowles, W. A. et al. Population-based study of antibody to the human polyomaviruses BKV and JCV and the simian polyomavirus SV40. J. Med. Virol. 71, 115-123 (2003).

3. Chatterjee, M., Weyandt, T. B. \& Frisque, R. J. Identification of archetype and rearranged forms of BK virus in leukocytes from healthy individuals. J. Med. Virol. 60, 353-362 (2000).

4. Heritage, J., Chesters, P. M. \& McCance, D. J. The persistence of papovavirus BK DNA sequences in normal human renal tissue. J. Med. Virol. 8, 143-150 (1981).

5. Egli, A. et al. Prevalence of polyomavirus BK and JC infection and replication in 400 healthy blood donors. J. Infect. Dis. 199, 837-846 (2009).

6. Acott, P. D. \& Hirsch, H. H. BK virus infection, replication, and diseases in pediatric kidney transplantation. Pediatr. Nephrol. 22, 1243-1250 (2007).

7. White, L. H. et al. BK virus nephropathy in renal transplant patients in London. Transplantation 85, 1008-1015 (2008).

8. Haysom, L. et al. BK viral infection in an Australian pediatric renal transplant population. Pediatr. Transplant. 8, 480-484 (2004).

9. Nickeleit, V. et al. Testing for polyomavirus type BK DNA in plasma to identify renal-allograft recipients with viral nephropathy. N. Engl. J. Med. 342, 1309-1315 (2000).

10. Randhawa, P. S. et al. Human polyoma virus-associated interstitial nephritis in the allograft kidney. Transplantation 67, $103-109$ (1999).

11. Wu, S. W., Chang, H. R. \& da Lian, J. The effect of low-dose cidofovir on the long-term outcome of polyomavirus-associated nephropathy in renal transplant recipients. Nephrol. Dial. Transplant. 24, 1034-1038 (2009).

12. Lee, B. T. et al. Efficacy of levofloxacin in the treatment of BK viremia: A multicenter, double-blinded, randomized, placebocontrolled trial. Clin. J. Am. Soc. Nephrol. 9, 583-589 (2014).

13. Santeusanio, A. D., Lukens, B. E. \& Eun, J. Antiviral treatment of BK virus viremia after kidney transplantation. Am. J. Health-Syst. Pharm. 74, 2037-2045 (2017). 
14. Brennan, D. C. et al. Incidence of BK with tacrolimus versus cyclosporine and impact of preemptive immunosuppression reduction. Am. J. Transplant. 5, 582-594 (2005).

15. Ginevri, F. et al. Prospective monitoring of polyomavirus BK replication and impact of pre-emptive intervention in pediatric kidney recipients. Am. J. Transplant. 7, 2727-2735 (2007).

16. Alexander, R. T., Langlois, V., Tellier, R., Robinson, L. \& Hébert, D. The prevalence of BK viremia and urinary viral shedding in a pediatric renal transplant population: A single-center retrospective analysis. Pediatr. Transplant. 10, 586-592 (2006).

17. Herman, J. et al. Polyomavirus infection in pediatric renal transplant recipients: Evaluation using a quantitative real-time PCR technique. Pediatr. Transplant. 8, 485-492 (2004).

18. Hymes, L. C. \& Warshaw, B. L. Polyomavirus (BK) in pediatric renal transplants: Evaluation of viremic patients with and without BK associated nephritis. Pediatr. Transplant. 10, 920-922 (2006).

19. Müller, A. et al. Detection of polyomavirus BK and JC in children with kidney diseases and renal transplant recipients. Pediatr. Infect. Dis. J. 24, 778-781 (2005).

20. Smith, J. M. et al. Polyomavirus nephropathy in pediatric kidney transplant recipients. Am. J. Transplant. 4, 2109-2117 (2004).

21. Höcker, B. et al. Epidemiology of and risk factors for BK polyomavirus replication and nephropathy in pediatric renal transplant recipients: An international CERTAIN registry study. Transplantation 103, 1224-1233 (2019).

22. Smith, J. M., Dharnidharka, V. R., Talley, L., Martz, K. \& McDonald, R. A. BK virus nephropathy in pediatric renal transplant recipients: An analysis of the North American Pediatric Renal Trials and Collaborative Studies (NAPRTCS) registry. Clin. J. Am. Soc. Nephrol. 2, 1037-1042 (2007).

23. Ginevri, F. et al. Polyomavirus BK infection in pediatric kidney-allograft recipients: A single-center analysis of incidence, risk factors, and novel therapeutic approaches. Transplantation 75, 1266-1270 (2003).

24. Reischig, T. et al. Cytomegalovirus prevention strategies and the risk of BK polyomavirus viremia and nephropathy. Am. J. Transplant. 19, 2457-2467 (2019).

25. Grenda, R. et al. A randomized trial to assess the impact of early steroid withdrawal on growth in pediatric renal transplantation: The twist study. Am. J. Transplant. 10, 828-836 (2010).

26. Hirsch, H. H. et al. Prospective study of polyomavirus type BK replication and nephropathy in renal-transplant recipients. N. Engl. J. Med. 347, 488-496 (2002).

27. Voigt, S., Rosario, A. S. \& Mankertz, A. Cytomegalovirus seroprevalence among children and adolescents in Germany: Data from the German Health Interview and Examination Survey for Children and Adolescents (KiGGS), 2003-2006. Open Forum Infectious Diseases 3, (2016).

28. Schwartz, G. J. \& Gauthier, B. A simple estimate of glomerular filtration rate in adolescent boys. J. Pediatr. 106, 522-526 (1985).

29. Höcker, B. et al. Epidemiology and morbidity of Epstein-Barr virus infection in pediatric renal transplant recipients: A multicenter. Prospective Study. https://doi.org/10.1093/cid/cis823 (2012).

30. Hirsch, H. H. \& Randhawa, P. BK virus in solid organ transplant recipients. Am. J. Transplant. 9, S136-S146 (2009).

\section{Acknowledgements}

We thank Prof. Rachel Lennon and Dr. Nick Plant for their advice during the preparation of this manuscript. The study was supported by a Jean Shanks/Pathological Society Clinical Lecturer Grant (Grant reference: JSPS CLG 2019 02) (awarded to JM).

\section{Author contributions}

J.M. and M.S. designed the study. J.M. and V.B. analysed data. All authors contributed to the preparation of the manuscript.

\section{Competing interests}

The authors declare no competing interests.

\section{Additional information}

Supplementary Information The online version contains supplementary material available at https:/doi. org/10.1038/s41598-021-82160-0.

Correspondence and requests for materials should be addressed to J.M.

Reprints and permissions information is available at www.nature.com/reprints.

Publisher's note Springer Nature remains neutral with regard to jurisdictional claims in published maps and institutional affiliations.

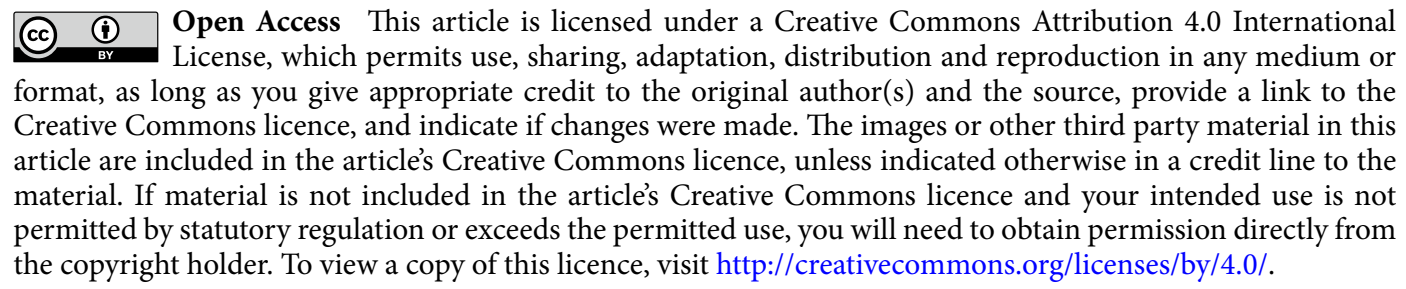

(C) The Author(s) 2021 